\title{
Contrasting determinants for the introduction and establishment success of exotic birds in Taiwan using decision trees models
}

\author{
Shih-Hsiung Liang ${ }^{1}$, Bruno Andreas Walther ${ }^{2}$, Bao-Sen Shieh ${ }^{\text {Corresp. } 3,4}$ \\ 1 Department of Biotechnology, National Kaohsiung Normal University, Kaohsiung, Taiwan \\ 2 Master Program in Global Health and Development, College of Public Health, Taipei Medical University, Taipei, Taiwan \\ 3 Department of Biomedical Science and Environmental Biology, Kaohsiung Medical University, Kaohsiung, Taiwan \\ 4 Department of Medical Research, Kaohsiung Medical University Hospital, Kaohsiung, Taiwan \\ Corresponding Author: Bao-Sen Shieh \\ Email address: bsshieh@kmu.edu.tw
}

Background. Biological invasions have become a major threat to biodiversity, and identifying determinants underlying success at different stages of the invasion process is essential for both prevention management and testing ecological theories. To investigate variables associated with different stages of the invasion process in a local region such as Taiwan, potential problems using traditional parametric analyses include too many variables of different data types (nominal, ordinal, and interval) and a relatively small data set with too many missing values. Methods. We therefore used five decision tree models instead and compared their performance. Our dataset contains 283 exotic bird species which were transported to Taiwan; of these 283 species, 95 species escaped to the field successfully (introduction success); of these 95 introduced species, 36 species reproduced in the field of Taiwan successfully (establishment success). For each species, we collected 22 variables associated with human selectivity and species traits which may determine success during the introduction stage and establishment stage. For each decision tree model, we performed three variable treatments: (I) including all 22 variables, (II) excluding nominal variables, and (III) excluding nominal variables and replacing ordinal values with binary ones. Five performance measures were used to compare models, namely, area under the receiver operating characteristic curve (AUROC), specificity, precision, recall, and accuracy. Results. The gradient boosting models performed best overall among the five decision tree models for both introduction and establishment success and across variable treatments. The most important variables for predicting introduction success were the bird family, the number of invaded countries, and variables associated with environmental adaptation, whereas the most important variables for predicting establishment success were the number of invaded countries and variables associated with reproduction. Discussion. Our final optimal models achieved relatively high 
performance values, and we discuss differences in performance with regard to sample size and variable treatments. Our results showed that, for both the establishment model and introduction model, the number of invaded countries was the most important or second most important determinant, respectively. Therefore, we suggest that future success for introduction and establishment of exotic birds may be gauged by simply looking at previous success in invading other countries. Finally, we found that species traits related to reproduction were more important in establishment models than in introduction models; importantly, these determinants were not averaged but either minimum or maximum values of species traits. Therefore, we suggest that in addition to averaged values, reproductive potential represented by minimum and maximum values of species traits should be considered in invasion studies. 
1 Contrasting determinants for the introduction and establishment success of exotic birds in

2 Taiwan using decision trees models

3

4

5 Shih-Hsiung Liang1, Bruno Andreas Walther ${ }^{2}$, Bao-Sen Shieh ${ }^{3,4 *}$

$6{ }^{1}$ Department of Biotechnology, National Kaohsiung Normal University, Kaohsiung, Taiwan;

$7{ }^{2}$ Master Program in Global Health and Development, College of Public Health, Taipei Medical

8 University, Taipei, Taiwan; ${ }^{3}$ Department of Biomedical Science and Environmental Biology,

9 Kaohsiung Medical University, Kaohsiung, Taiwan; ${ }^{4}$ Department of Medical Research,

10 Kaohsiung Medical University Hospital, Kaohsiung, Taiwan

11

12 Corresponding author:

13 Bao-Sen Shieh

14 e-mail address: bsshieh@kmu.edu.tw

15 Tel: 886-7-3121101ext.2703

16 Fax: 886-7-3227508

17 


\section{Abstract}

19 Background. Biological invasions have become a major threat to biodiversity, and

20 identifying determinants underlying success at different stages of the invasion process is

21 essential for both prevention management and testing ecological theories. To investigate

22 variables associated with different stages of the invasion process in a local region such as Taiwan,

23 potential problems using traditional parametric analyses include too many variables of different

24 data types (nominal, ordinal, and interval) and a relatively small data set with too many missing 25 values.

26 Methods. We therefore used five decision tree models instead and compared their

27 performance. Our dataset contains 283 exotic bird species which were transported to Taiwan; of

28 these 283 species, 95 species escaped to the field successfully (introduction success); of these 95

29 introduced species, 36 species reproduced in the field of Taiwan successfully (establishment

30 success). For each species, we collected 22 variables associated with human selectivity and

31 species traits which may determine success during the introduction stage and establishment stage.

32 For each decision tree model, we performed three variable treatments: (I) including all 22

33 variables, (II) excluding nominal variables, and (III) excluding nominal variables and replacing

34 ordinal values with binary ones. Five performance measures were used to compare models,

35 namely, area under the receiver operating characteristic curve (AUROC), specificity, precision,

36 recall, and accuracy.

37 Results. The gradient boosting models performed best overall among the five decision tree

38 models for both introduction and establishment success and across variable treatments. The most

39 important variables for predicting introduction success were the bird family, the number of

40 invaded countries, and variables associated with environmental adaptation, whereas the most 
41 important variables for predicting establishment success were the number of invaded countries

42 and variables associated with reproduction.

43 Discussion. Our final optimal models achieved relatively high performance values, and we

44 discuss differences in performance with regard to sample size and variable treatments. Our

45 results showed that, for both the establishment model and introduction model, the number of

46 invaded countries was the most important or second most important determinant, respectively.

47 Therefore, we suggest that future success for introduction and establishment of exotic birds may

48 be gauged by simply looking at previous success in invading other countries. Finally, we found

49 that species traits related to reproduction were more important in establishment models than in

50 introduction models; importantly, these determinants were not averaged but either minimum or

51 maximum values of species traits. Therefore, we suggest that in addition to averaged values,

52 reproductive potential represented by minimum and maximum values of species traits should be

53 considered in invasion studies.

\section{4}

55

56 


\section{Introduction}

58 Biological invasions have become a major threat to biodiversity (Pimentel, Zuniga \&

59 Morrison, 2005). Hence, some studies of biological invasion have focused on how to prevent the 60 invasion or how to eradicate the invasive species (Dana, Jeschke \& Garcia-de-Lomas, 2014). As

61 more and more invasive species have spread into the wild, invasive species have also become

62 important subjects in testing ecological theories in relation to niche and competition (e.g.,

63 Broennimann et al., 2007; Allen et al., 2015). Both prevention management and testing

64 ecological theories require the identification of the key factors underlying success at different

65 stages in the invasion process (Duncan, Blackburn \& Sol, 2003); moreover, factors that are

66 important to explain the invasion success have been suggested to be different at each stage of the

67 invasion process (Kolar \& Lodge, 2002; Williamson, 2006; Dawson, Burslem \& Hulme, 2009).

68 Compared with other vertebrate taxa, birds have a higher number of invasive species and

69 invasion success rates in a study focusing on Europe and North America (Jeschke \& Strayer,

70 2006). Previous studies on exotic birds have identified two major categories of factors associated

71 with their success at the introduction and establishment stages: human selectivity factors and

72 species traits. Human selectivity factors consist of factors such as taxa and geography selected

73 non-randomly by humans during the transport or introduction stages of exotic birds (Duncan,

74 Blackburn \& Sol, 2003). Species traits, on the other hand, then play an important role during the

75 introduction and establishment stages (Blackburn, Cassey \& Lockwood, 2009).

76 In Taiwan, at least 290 exotic species of pet birds have been imported, and a 9.7\% rate of

77 invasion success was estimated (Shieh et al., 2006). For the transport stage, non-random

78 selectivity of exotic birds imported to Taiwan was associated with bird family, native geographic 79 range, body size, and song production of species (Su, Cassey \& Blackburn, 2014); as to the later 
80 stages of invasion, pet trade factors such as song attractiveness were significantly associated with

81

82

83 introduction success but not establishment success (Su, Cassey and Blackburn, 2016).

For the exotic birds of Taiwan, species traits that help to avoid stochastic extinction or to constrain establishment (cf. Sol, 2008) have not been investigated with regard to their influences on different stages of the invasion process. To investigate the effects of these factors which are associated with both human selectivity and species traits onto different stages of the invasion process in a local region such as Taiwan, two potential problems using traditional parametric analyses have been identified as (1) a relatively small data set with too many missing values and (2) too many variables of different types (nominal, ordinal, and interval).

Machine learning is a new, advanced analytical method which overcomes many of the restrictions of traditional parametric analyses. We chose the decision tree method, a machine learning algorithm, because its advantages include no need to input data for missing values and no assumptions about the distribution of the data; therefore, this method is ideal for dealing with mixed data types, such as nominal, ordinal and interval variables (Olinsky, Kennedy \& Brayton Kennedy, 2014). In studies of biological invasion, the decision tree method was first applied to investigating a data set of 45 fish species for risk assessment in the Great Lakes (Kolar \& Lodge, 2002). In another recent study, Chen, Peng \& Yang (2015) found that decision tree methods not only work best with nominal variables but also have higher performance values than traditional parametric methods in predicting alien herb invasion. In a comparative study of trait-based risk assessment for invasive species which included a bird data set, Keller, Kocev \& Džeroski (2011) found that random forests (an ensemble method that creates multiple decision tree sub-models) was one of the two best performing methods. Vall-llosera \& Sol (2009) investigated only one of the four stages of the invasion process, namely establishment success, in a global risk assessment for invasive birds. They found that their tree model had an overall predictive accuracy as high as 
104 the conventional statistical models (generalized linear mixed models). Besides these two studies, 105 which only focused on the establishment stage for exotic birds using decision tree models, there

106 are, to our knowledge, no other studies which used decision tree methods to analyze the

107 determinants for both the introduction and establishment stages of exotic birds.

108 Consequently, we decided to use decision tree methods to assess factors associated with

109 human selectivity and species traits which determine the success during the introduction and

110 establishment stages of exotic birds in Taiwan. We used five decision tree models which differed

111 in regard to resampling the data set and compared their performance. An optimal prediction

112 model was chosen based on five performance measures, and the relative importance of factors in

113 the optimal model for introduction success and for establishment success was examined and 114 compared.

115

116 Materials \& Methods

117 Species of the data set

118 The four stages of the invasion process were defined in Duncan, Blackburn \& Sol (2003) as

119 transport, introduction, establishment, and spread. In this study, we focused on the introduction

120 and establishment stages. For a species to reach the introduction stage, it must have passed the

121 transport stage. Therefore, we selected all the exotic species which had been transported to

122 Taiwan's main island (not including surrounding islands, such as Lanyu and Kinmen Island) as

123 documented in Shieh et al. (2006) which included the results of Chi (1995), Severinghaus (1999)

124 and Lin (2004). Whether a transported species has passed the subsequent stages of the invasion

125 process was based (1) on escaping records in the field (introduction success) and (2) breeding

126 record in the field (establishment success). We followed the detailed methods of how to define

127 introduction success and establishment success which were given in Su, Cassey and Blackburn 
128 (2016). However, Su, Cassey and Blackburn (2016) based their decision of establishment

129 success on the respective species having been recorded to be breeding at least twice; instead, we

130 based it on at least one record of fledglings actually having left the nest successfully.

131 In order to record all the escaping and breeding records of bird species up to 2015, we

132 continuously (1) checked information from the Chinese Wild Bird Federation

133 (http://www.bird.org.tw/) database which is the main collector of wild bird data in Taiwan, as

134 well as other Taiwanese websites dedicated to natural history observations of birds, (2) remained 135 in contact with local ornithologists, birdwatchers and bird societies, and (3) included any 136 relevant publications (e.g., Walther, 2011; Walther 2014 for red-whiskered bulbul, Pycnonotus

137 jocosus; Fan et al., 2009 for white-rumped shama, Copsychus malabaricus, or Shieh, Lin \&

138 Liang, 2016 for Asian glossy starling, Aplonis panayensis). Most of this updated information was 139 published recently in a project report for the Taiwan Forestry Bureau (Liang \& Shieh, 2016).

140 Despite following the methods as described in Su, Cassey and Blackburn (2016), we

141 independently collected all the data used in this analysis beginning in 2004 and ending in 2015.

142 Our dataset thus contains 283 full species (although we entered subspecies in our dataset, for this

143 analysis, we only used full species) which were transported to Taiwan (see above). Of these 283

144 species, 95 species escaped to the field successfully (introductions success). Of these 95 species,

14536 species reproduced in the field of Taiwan successfully (establishment success) (see

146 supplementary file Table S1 for species list).

147

148 Variables

149 We collected 22 variables for each species, including two nominal ones (order and family

150 taxa), six ordinal ones (latitude overlap with Taiwan: $0-2$, migration pattern: $0-3$, nesting location:

151 0-3, feeding: 1-3, diet: 1-6, and habitat: 0-6), three binary ones (hole nest, Taiwan genus_resident, 
152 dichromatism), and 11 interval ones (clutch size: Clutch, maximum clutch size: Mclutch,

153 incubation days: Incubation, minimum incubation days: Minincubation, body length: Length,

154 maximum body length: Mlength, body mass: Mass, maximum body mass: Mmass, the number of

155 invaded countries: Invcountry_Max, distribution range $\left(\mathrm{km}^{2}\right)$ : Range, the number of subspecies:

156 subspecies) (see supplementary file Table S2 for code descriptions of variables). The variable

157 Taiwan genus_resident was based on the information in Hsiao \& $L i$ (2014). For the other

158 variables, we gathered species information from the books of del Hoyo et al. (1992-2011),

159 Dunning Jr. (1993), and internet databases of IUCN (www.iucn.org) and BirdLife International

160 Datazone (http://datazone.birdlife.org) (see supplementary file Table S1 for associated

161 information of each species and Table S2 for code descriptions of variables). When we collected

162 the values for reproduction and body size for each species, we usually found a given range

163 instead of fixed values in the references. In order to account for the maximum adaptation and

164 reproduction potential in the invasion process, we used maximum values such as maximum body

165 mass or minimum values such as minimum incubation days in addition to averaged values. To

166 determine the number of invaded countries (Invcountry_Max), we counted the total (or

167 maximum) number of countries in which occurrences of introduced populations of each

168 respective species were reported.

169

170 Decision trees models and variable treatments

171 To investigate the possible effects of nominal variables (family and order) and ordinal

172 variables on the performance of the decision tree models, we conducted three variable treatments

173 for modeling: (I) including all variables, (II) excluding nominal variables, and (III) excluding

174 nominal variables and replacing ordinal values with binary ones; e.g. changing habitat values of

175 0-4 to 0 (natural habitats) and habitat values of 5-6 to 1 (artificial habitats). 
176 For each variable treatment, we used five decision tree models (DT_no bagging, DT_bagging

177 90\%, DT_bagging 100\%, gradient boosting, and HP forest) to predict the outcomes of

178 introduction and establishment, respectively. Modeling processes and comparisons of model

179 performance were implemented using SAS Enterprise Miner 13.1 (for diagrams of process flow,

180 see supplementary files Fig. S1 \& Fig. S2). Because of the small data set, no data partition was

181 implemented; that is, all data were used as training data. Instead, other methods, such as bagging

182 and cross validation, which have been suggested for the use with small data sets (SAS Institute

183 Inc., 2013), were used in the present study.

184 DT_no bagging is the traditional classification tree method by constructing a layered tree

185 model with the following settings: splitting rule = Gini, cross validation with 10 subsets and 100

186 repeats. The DT_bagging 90\% and DT_bagging 100\% methods used the same setting of splitting

187 rule and cross validation as the DT_no bagging method but with bagging $90 \%$ or $100 \%$ of the

188 data set for 50 times, respectively. Gradient boosting is a boosting method that resamples the

189 data set to produce a series of decision trees which together form a single predictive model which

190 has been found to be less prone to overfitting the data than a single decision tree (Georges, 2009).

191 HP Forest is the random forest method which builds many parallel trees forming a forest; a tree

192 in the forest is a sample without replacement from all the available observations, and the input

193 variables that are considered for splitting a node are randomly selected from all the available

194 inputs (Hall et al., 2014).

195 We calculated five performance measures to compare models, namely, the area under the 196 receiver operating characteristic curve (AUROC), the specificity which measures the fraction of

197 negative events that were correctly labeled, the precision which measures the fraction of

198 positively labeled outcomes that were correctly labeled, the recall which measures the fraction of

199 positive events that were correctly labeled, and the accuracy which measures the fraction of all 
200 events that were correctly labeled (accuracy $=1$ - misclassification rate) (Sohngen, Chang \& 201 Schomburg, 2011). These five performance measures have the same range (0-1), and we gave 202 each measure equal weight in evaluating the model performance in accordance with Chen, Peng $203 \&$ Yang (2015). The higher the values of these five performance measures are, the better the 204 model performs; therefore, we summed up the five values (called the "total score" from 205 hereupon) and chose the model with the highest sum as our final optimal model. We then 206 compared the relative importance of each of the variables in the optimal introduction model and 207 establishment model.

208 For illustrative purposes, we chose the visual output of the resulting trees of DT_no bagging 209 of variable treatment I for our figures (Fig. 1 \& Fig. 2). Such visual outputs are not possible for 210 the other four methods (namely, DT_bagging 90\%, DT_bagging 100\%, gradient boosting, and 211 HP forest).

212 We used the decision tree models described above to build various versions of two kinds of 213 models: (1) introduction success prediction models and (2) establishment success prediction 214 models. However, for brevity's sake, from hereon we will call them introduction models and 215 establishment models, respectively.

217 Results

218 Across the three variable treatments and for both the introduction models (Table 1) and 219 establishment models (Table 2), the gradient boosting models always achieved the highest score 220 among the five decision tree models (i.e., it performed best overall). However, this overall best

221 performance did not mean that gradient boosting always performed best when comparing values 222 of the five performance measures. For instance, Table 1 (see also supplementary file Fig. S3 for 223 receiver operating characteristic curves, and supplementary file Fig. S4 for classification charts) 
224 shows that gradient boosting only performed best for accuracy in variable treatment I and II;

225 otherwise, other models always performed better using the other four performance measures.

226 Nevertheless, across all three treatments, the total score is always highest for gradient boosting

227 for the introduction models (Table 1).

228 For the establishment models (Table 2, see also supplementary file Fig. S5 for receiver

229 operating characteristic curves, and supplementary file Fig. S6 for classification charts), however,

230 gradient boosting has the highest total score for all the three treatments and also for most of the

231 five performance measures (the only exceptions being specificity and precision in variable

232 treatment II). Therefore, we considered gradient boosting the optimal model for both the

233 introduction models and establishment models and only considered its results from hereupon.

234 Looking across the three different variable treatment methods I-III, gradient boosting

235 performed best with variable treatment I for the introduction models (Table 1) as well as the

236 establishment models (Table 2). For variable treatments II and III, the total score decreased by

237 only $0.169(4 \%)$ and $0.128(3 \%)$, respectively. We also note that this decreasing trend across

238 variable treatments is maintained for most of the five performance measures. Furthermore, the

239 values of the performance measures are all $>0.7$ and $60 \%$ are $>0.9$, which means that the

240 performance was consistently high or very high.

241 In the optimal introduction model, family and the number of invaded countries

242 (Invcountry_Max) were the most important variables, and their relative importance values were

2431 and 0.888 , respectively (Fig. 3). The top six variables with an importance value $>0.3$ also

244 included maximum body mass (0.394), order (0.384), latitude overlap with Taiwan (0.354), and

245 distribution range (0.345). For the introduction model based on the classification tree method

246 (Fig. 1), the number of invaded countries was the most important determinant, as it appeared at

247 the top of the tree, which means that the 84 species with any record of invading other countries 
248 had a $66.7 \%$ chance of successful introduction to Taiwan. Among these 84 species, the 72

249 species which had a migration pattern categorized as sedentary (0), local movement (1) or partial

250 migration (2) had a $73.6 \%$ chance of successful introduction, while the 12 species categorized as

251 migrants (3) had only a $25.0 \%$ chance of successful introduction. Among the 199 species which

252 had no record of invading other countries, family was chosen as an important determinant of 253 successful introduction.

254 In the optimal establishment model, the number of invaded countries and distribution range 255 were the most important variables, and their relative importance values were 1 and 0.826 , 256 respectively (Fig. 3). The top six variables with an importance value $>0.6$ also included 257 minimum incubation days (Minincub, 0.647), migration pattern (Migration, 0.633), clutch size 258 (Clutch, 0.62), and habitat type (Habitat, 0.616). The relative importance of the variable family 259 decreased to 0.569 which is therefore much lower than in the optimal introduction model (see 260 above). For the establishment model based on the classification tree method (Fig. 2), the number 261 of invaded countries was again the most important determinant, as it appeared at the top of the 262 tree. However, in this case it means that the 39 species with a record of invading at least two 263 countries had a $59.0 \%$ chance of successful establishment in Taiwan, while the 56 species with a 264 record of invading fewer than two countries had only a $23.2 \%$ chance of successful 265 establishment. Among the 39 species noted above, the 21 species with a maximum clutch size 266 (Mclutch) $<5.5$ had an $81.0 \%$ chance of successful establishment, while the other 18 with a 267 maximum clutch size of $\geq 5.5$ had only a $33.3 \%$ chance of successful establishment. Finally, 268 among the 56 species noted above, the eight species with a body length (Length) $\geq 36.5 \mathrm{~cm} \mathrm{had} \mathrm{a}$ $26962.5 \%$ chance of successful establishment.

271 Discussion 
272 Model comparisons and variable treatment comparisons

273 Our results showed that for the complete data set of 283 transported species or for the data

274 set of 95 introduced species, the gradient boosting method performed better than the other four

275 decision tree methods. While we calculated five performance measures, the only other study

276 which used the decision tree method on a bird data set was Keller, Kocev \& Džeroski (2011) who

277 calculated only AUROC and accuracy values. Considering AUROC values first, the AUROC

278 values of gradient boosting of our study were over 0.919 in the introduction models and over

2790.971 in the establishment models; thus, they were all higher than our values for the random

280 forests method. This is in contrast to the results of Keller, Kocev \& Džeroski (2011) who found

281 that, based on the AUROC values, random forests performed better than gradient boosting for

282 both their New Zealand and Australia bird data sets. Specifically, AUROC values for gradient

283 boosting for their New Zealand (79 species with 11 traits) and Australia (52 species with 11 traits)

284 data sets were 0.682 and 0.681 , respectively, whereas AUROC values for random forests were

2850.731 and 0.745, respectively. Pearce \& Ferrier (2000) suggested that AUROC values between

2860.7 and 0.9 indicate a reasonable discrimination ability of models, and values higher that 0.9

287 indicate a very good discrimination ability of models. The higher AUROC values of our study

288 might have resulted from the inclusion of more variables (up to 22 variables) rather than larger

289 samples used for analysis. In our study, both the introduction model and establishment model

290 used 22 variables, and we found higher AUROC values (0.971-0.985) in the smaller data set

291 (namely, the establishment model with 95 species) than in the larger data set (namely, the

292 introduction model with 283 species) (AUROC values 0.919-0.936). We therefore suggest that

293 even a small data set (less than 100 species) with up to 22 variables can achieve a prediction

294 model of good performance using the gradient boosting method.

295 Comparing the performances of variable treatment I with variable treatments II and III, we 
296 found little difference on model performance. Treatment II excluded nominal variables, and

297 treatment III changed ordinal variables of species traits into binary variables, but neither one of

298 these changes really had much discernable influence on overall performance. Our results

299 therefore provided evidence to support the use of ordinal variables of species traits, and that there

300 is no need to convert ordinal variables of species traits to binary ones for their use in decision

301 tree models.

302

303 Predictors of introduction and establishment success in exotic birds

304 Perhaps the most interesting and novel result of our study is that, for both the establishment

305 model and introduction model, the number of previously invaded countries was the most

306 important or second most important determinant in all the models. Therefore, our study suggests

307 that future success for introduction and establishment of birds can be gauged by simply looking

308 at previous success in invading other countries or regions. Future studies should include this

309 variable to confirm our supposition because it might be a very simple and straightforward way to

310 predict the potential invasion success of a species: if it has been successful before, it will

311 probably be successful again. While this variable could not have been established a few decades

312 ago, we now have a global track record of successful species invasions, and we might therefore

313 be able to use it to better predict future local or regional invasions. Furthermore, global studies

314 could investigate what species traits and other relevant factors, e.g., local ecological factors, are

315 related to the number of successfully invaded countries; or, given the differential size of

316 countries, the actual area invaded.

317 Another important determinant was family taxon. While family was the most important

318 variable in the optimal introduction model, it dropped to being only the seventh most important

319 variable in the optimal establishment model. In other words, family was an important 
320 determinant of introduction but not establishment in Taiwan. Our results thus differ from those

321 of a global study which found that bird family was also a good predictor for establishment

322 success (Lockwood 1999). The discrepancy between this study and our study could result from

323 the fact that exotic birds in Taiwan are primarily introduced for aesthetic reasons but not for

324 hunting (Shieh et al., 2006; Su, Cassey \& Blackburn, 2016), while the global data set included

325 many hunted species.

326 Several species traits were also chosen as important determinants for the introduction and 327 establishment models. For the optimal introduction model, the top three selected species traits 328 were maximum body mass (Mmass), latitude overlap with Taiwan (Overlap), and distribution 329 range (Range). Among these three variables, maximum body mass was ranked the most 330 important, and it also had a relative importance greater than that of two other closely related 331 measures, specifically, the averaged body mass (Mass) and body length (Length). One possible 332 explanation is that birds are usually heavier in captivity under well fed condition. Our data set 333 contained primarily pet species (and not game species, which are prevalent in many other

334 studies), and the body mass of pet birds might be higher than the average body mass of their wild 335 congeners, and therefore closer to the maximum attainable body mass. In order to consider the 336 representability and the maximum adaptation potential in the invasion process, we therefore 337 suggest that including maximum body mass may be important in order not to miss a potentially 338 important determinant for the invasion success of exotic pet birds in particular. For example, $\mathrm{Su}$,

339 Cassey \& Blackburn (2016) did not find that body mass had any influence on introduction 340 success. However, they only used averaged body mass, and perhaps their result would have been 341 different if they had also included maximum body mass. Furthermore, Cassey's (2001) global 342 study found that averaged body mass was significantly correlated with introduction success 
343 which further supports the role of some measure of body mass being an important determinant of

344 introduction success.

345 Finally, several species traits related to reproduction were also important, such as minimum

346 incubation days (Minincub), clutch size (Clutch), dichromatism, and nesting locations (Nesting);

347 however, these determinants were more important in establishment success than in introduction

348 success. Furthermore, given that some top ranking variables were associated with maximum or

349 minimum values of species traits, we suggest that in addition to averaged values, reproductive

350 potential represented by minimum and maximum values of species traits should be considered in

351 prediction models of invasion studies.

352 We conclude that decision tree models are efficient for the analysis of small data sets with

353 mixed types of variables, including nominal, ordinal and interval variables in predicting the

354 invasion success of exotic birds. Our results further demonstrate that the most important

355 determinants in predicting introduction success of exotic birds in Taiwan were the bird family,

356 the number of invaded countries, and variables associated with environmental adaptation,

357 whereas the most important determinants in predicting establishment success were the number of

358 invaded countries and variables associated with reproduction.

359

360 ACKNOWLEDGEMENTS

361 We thank Liviu Parau and two anonymous reviewers for their valuable comments and

362 suggestions on previous drafts of the manuscript.

363

364 Data availability

365 File: Supplementary S1 (Table S1-S2).xls

366 Table S1. Species list and associated variable information 
367 Table S2. Code description of variable

368

369 Supplemental Information

370 File: Supplementary S2 (Figure S1).png

$371 \quad$ Supplementary S3 (Figure S2).png

$372 \quad$ Supplementary S4 (Figure S3-S6).pdf

373 Figure S1. Diagrams of modeling introduction success using SAS Enterprise Miner: (a) variable 374 treatment I, (b) variable treatment II, and (c) variable treatment III.

375 Figure S2. Diagrams of modeling establishment success using SAS Enterprise Miner: (a)

376 variable treatment I, (b) variable treatment II, and (c) variable treatment III.

377 Figure S3. Receiver operating characteristic (ROC) curves of five introduction models: (a) 378 variable treatment I, (b) variable treatment II, and (c) variable treatment III.

379 Figure S4. Classification charts of five introduction models: (a) variable treatment I, (b) variable 380 treatment II, and (c) variable treatment III.

381 Figure S5. Receiver operating characteristic (ROC) curves of five establishment models: (a) variable treatment I, (b) variable treatment II, and (c) variable treatment III.

383 Figure S6. Classifications charts of five establishment models: (a) variable treatment I, (b) 384 variable treatment II, and (c) variable treatment III.

385

386

\section{References}

387 Allen CR, Angeler DG, Moulton MP, Holling CS. 2015. The importance of scaling for detecting 388 community patterns: success and failure in assemblages of introduced species. Diversity 7: $389 \quad 229-241$. 
390 Blackburn TM, Cassey P, Lockwood JL. 2009. The role of species traits in the establishment 391 success of exotic birds. Global Change Biology 15: 2852-2860.

392 Broennimann O, Treier UA, Müller-Schärer H, Thuiller W, Peterson AT, Guisan A. 2007. 393 Evidence of climatic niche shift during biological invasion. Ecology letters 10: 701-709. 394 Cassey P. 2001. Are there body size implications for the success of globally introduced land 395 birds? Ecography 24: 413-420.

396 Chen L, Peng S, Yang B. 2015. Predicting alien herb invasion with machine learning models: 397 biogeographical and life-history traits both matter. Biological Invasions 17: 2187-2198. 398 Chi W-L. 1995. An investigation report on pet bird trade in Taiwan. Green Consumer's 399 Foundation. Taipei, Taiwan. (In Chinese)

400 Dana ED, Jeschke JM, García-de-Lomas J. 2014. Decision tools for managing biological 401 invasions: existing biases and future needs. Oryx 48: 56-63.

402 Dawson W, Burslem DF, Hulme PE. 2009. Factors explaining alien plant invasion success in a 403 tropical ecosystem differ at each stage of invasion. Journal of Ecology 97: 657-665.

404 del Hoyo J, Elliott A, Sargatal J, Christie DA. 1992-2011. Handbook of the birds of the world, 405 vol. 1-16. Barcelona: Lynx Edicions.

406 Duncan RP, Blackburn, TM, \& Sol D .2003. The ecology of bird introductions. Annual Review 407 of Ecology, Evolution, and Systematics 71-98.

408 Dunning Jr, JB. 1993. CRC handbook of avian body masses. CRC press, Boca Raton, Florida. 409 Fan M-W, Lin R-S, Fang W \& Lin Y-H. 2009. The distribution and abundance of the alien 410 invasive white-rumped shama (Copsychus malabaricus) in Taiwan. Taiwania 54: 248-254.

411 Hall P, Dean J, Kabul IK, Silva J. 2014. An Overview of Machine Learning with SAS®

412 Enterprise Miner ${ }^{\mathrm{TM}}$. Cary: SAS Institute Inc. 
413 Hsiao M-C, Li C-L. 2014. Field Guide of Wild Bird in Taiwan. Taipei: Wild Bird Society of 414 Taiwan.

415 Jeschke JM, Strayer DL. 2006. Determinants of vertebrate invasion success in Europe and North 416 America. Global Change Biology 12: 1608-1619.

417 Keller RP, Kocev D, Džeroski S. 2011. Trait-based risk assessment for invasive species: high 418 performance across diverse taxonomic groups, geographic ranges and machine 419 learning/statistical tools. Diversity and Distributions 17: 451-461.

420 Kolar CS, Lodge DM. 2002. Ecological predictions and risk assessment for alien fishes in North 421 America. Science 298(5596): 1233-1236.

422 Liang S-H, Shieh B-S. 2016. Application and analysis of the life history database to assess 423 invasion impact of exotic animals (in Chinese with English abstract). Available at 424 http://conservation.forest.gov.tw/File.aspx?fno $=61675$.

425 Lin R-S. 2004. The black terrors of Hitchcock - current status of invasive birds in Taiwan. $426 \quad$ Nature Conservation Quarterly 48: 38-43. (In Chinese)

427 Lockwood JL (1999). Using taxonomy to predict success among introduced avifauna: relative 428 importance of transport and establishment. Conservation Biology 13(3): 560-567.

429 Olinsky A, Kennedy K, Brayton Kennedy B. 2014. Assessing gradient boosting in the reduction 430 of misclassification error in the prediction of success for actuarial majors. Case Studies In 431 Business, Industry And Government Statistics 5: 12-16.

432 Pearce J, Ferrier S. 2000. Evaluating the predictive performance of habitat models developed 433 using logistic regression. Ecological modelling 133: 225-245.

434 Pimentel D, Zuniga R, Morrison D. 2005. Update on the environmental and economic costs 435 associated with alien-invasive species in the United States. Ecological economics 52: 273-288. 
436 SAS Institute Inc. 2013. SAS® Enterprise Miner ${ }^{\mathrm{TM}}$ 13.1 Extension Nodes: Developer's Guide.

437 Cary: SAS Institute Inc.

438 Severinghaus L-L. 1999. Exotic birds in Taiwan. Wild Birds 7: 45-58.

439 Shieh B-S, Lin Y-H, Lee T-W, Chang C-C, Cheng K-T. 2006. Pet trade as sources of introduced 440 bird species in Taiwan. Taiwania 51: 81-86.

441 Shieh B-S, Lin C-J, Liang S-H. 2016. Breeding biology of the invasive Asian Glossy Starling 442 (Aplonis panayensis) in urban parks of Kaohsiung City, southern Taiwan. Taiwan J For Sci $443 \quad 31: 63-70$.

444 Söhngen C, Chang A, Schomburg D. 2011. Development of a classification scheme for disease445 related enzyme information. BMC bioinformatics 12: 329.

446 Sol D. 2008. Do successful invaders exist? Pre-adaptations to novel environments in terrestrial 447 vertebrates. In Biological invasions (pp. 127-141). Springer Berlin Heidelberg.

448 Su S, Cassey P, Blackburn TM. 2014. Patterns of non-randomness in the composition and 449 characteristics of the Taiwanese bird trade. Biological Invasions 16: 2563-2575.

450 Su S, Cassey P, Blackburn TM. 2016. The wildlife pet trade as a driver of introduction and 451 establishment in alien birds in Taiwan. Biological Invasions 18: 215-229.

452 Vall-llosera M, Sol D. 2009. A global risk assessment for the success of bird 453 introductions. Journal of Applied Ecology 46: 787-795.

454 Walther, B.A. (2011) First documented nesting of the red-whiskered bulbul Pycnonotus jocosus 455 in Taiwan. TW J. Biodivers 13: 121-133.

456 Walther, B.A. (2014) Second documented nesting of the red-whiskered bulbul Pycnonotus 457 jocosus in Taiwan. Feather 266: 20-25.

458 Williamson, M. (2006). Explaining and predicting the success of invading species at different 459 stages of invasion. Biological Invasions 8: 1561-1568. 


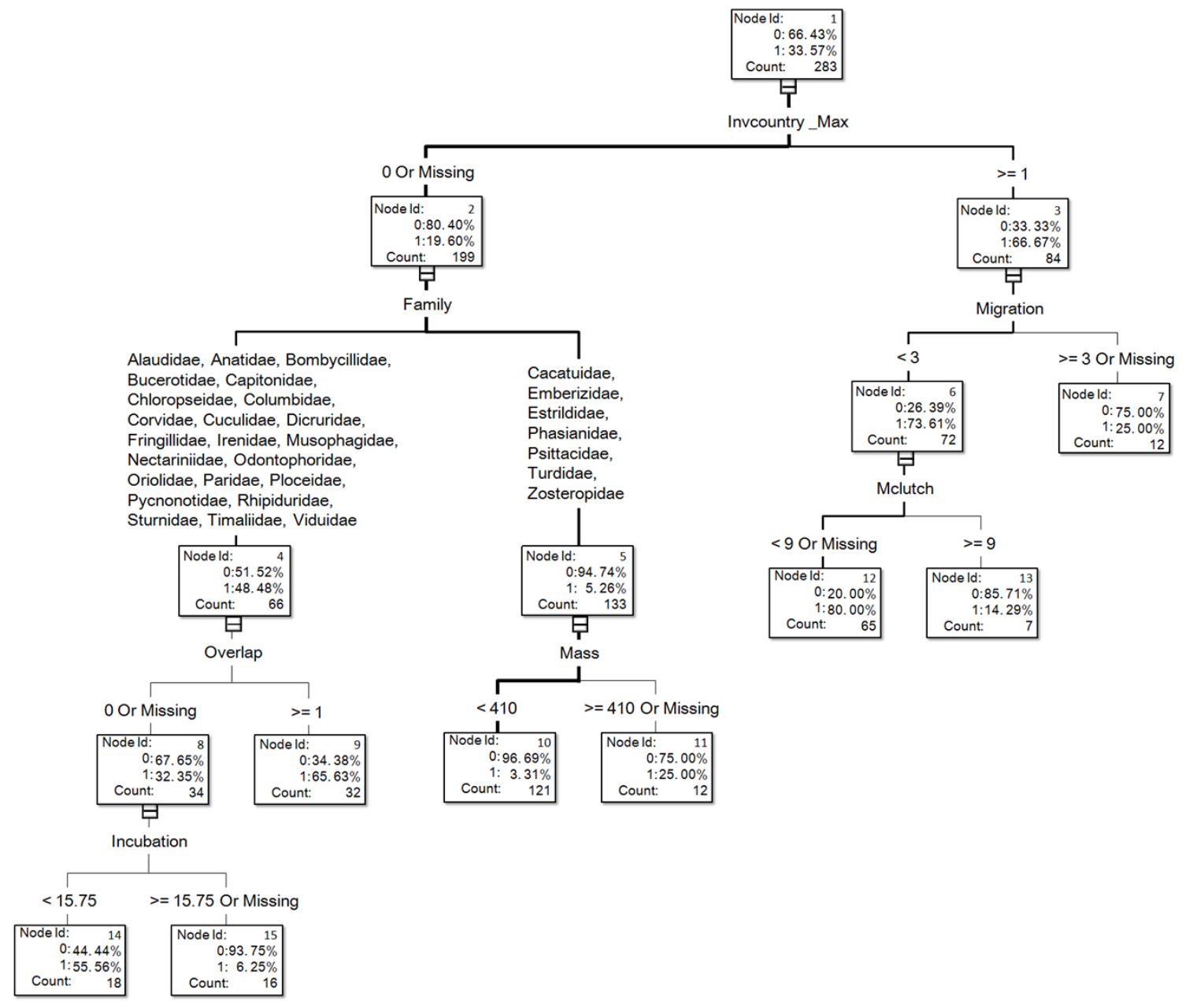

461

462

463 Fig. 1. The visual output of the introduction model based on the classification tree method for exotic birds of Taiwan generated from the dataset of 283 transported species, of which 95 species successfully escaped in the field (see supplementary file Table S1 for associated information of each species and Table S2 for code descriptions of variables)

467

468

469 


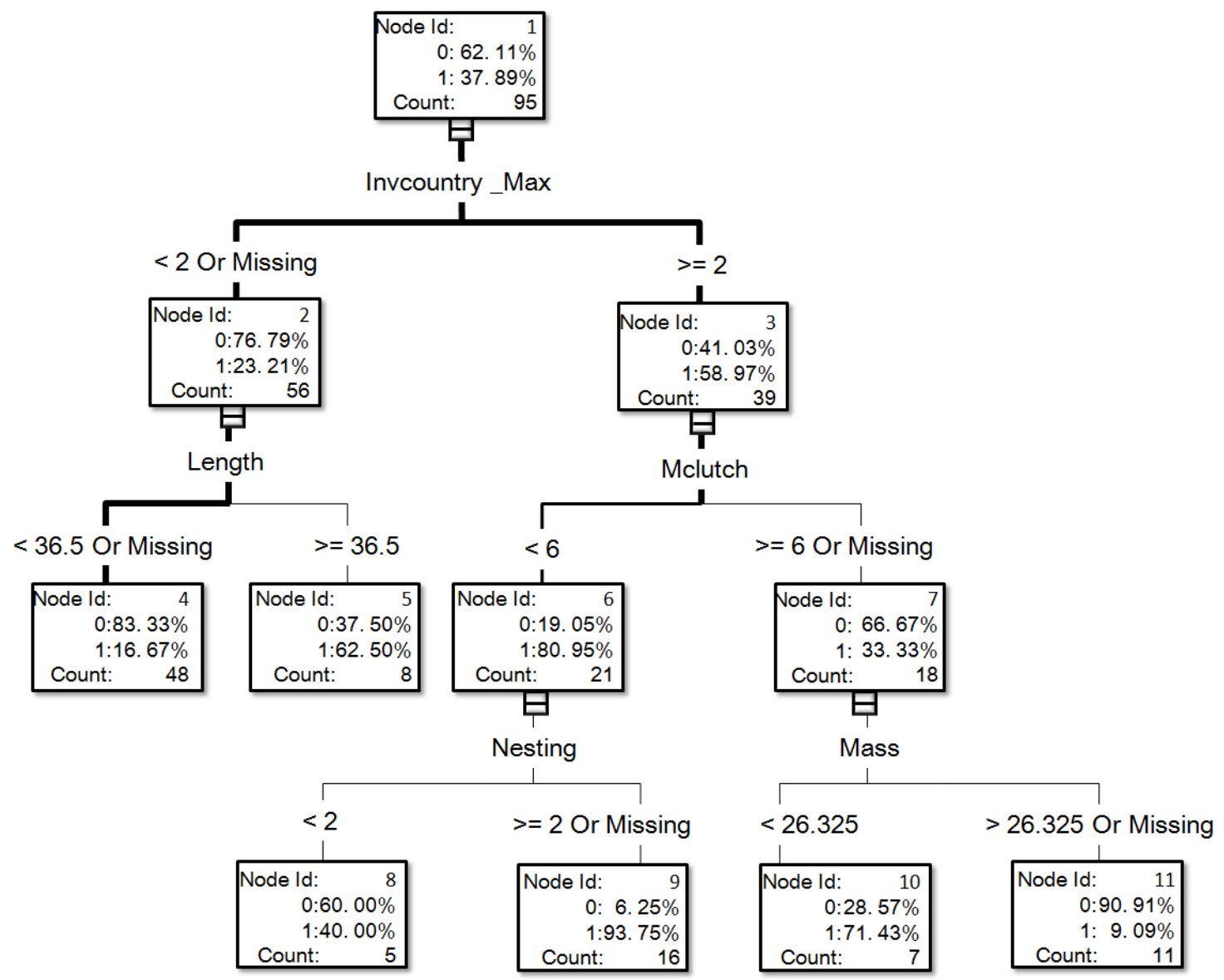

470

471

472 Fig. 2. The visual output of the establishment model based on the classification tree method for

473

474

475

476 exotic birds of Taiwan generated from the dataset of 95 introduced species, of which 36 species successfully reproduced in the field (see supplementary file Table S1 for associated information of each species and Table S2 for code descriptions of variables) 


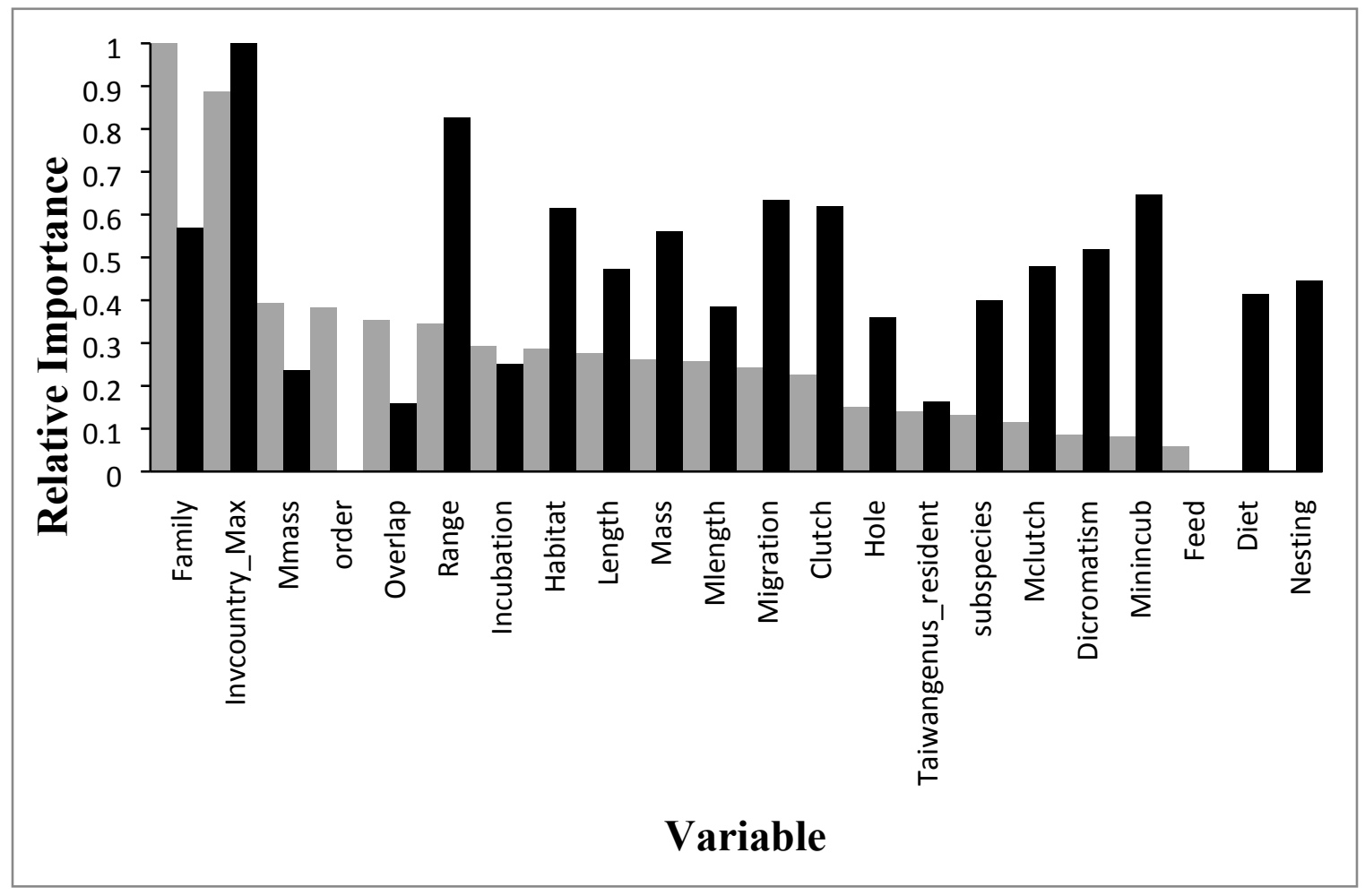

478

479 Fig. 3. Relative importance of variables in the prediction models using gradient boosting approach (grey bars for introduction models and black bars for establishment models).

481 For descriptions of codes for variables, see supplementary Table S2.

482 
483 Table 1. Comparison of five performance measures among five introduction models of exotic birds in Taiwan, separately for three variable treatments (see Methods for details).

Variable Treatment I

\begin{tabular}{|c|c|c|c|c|c|c|}
\hline Model & AUROC & Specificity & Precision & Recall & Accuracy & Total \\
\hline DT_no bagging & 0.894 & 0.830 & 0.722 & 0.874 & 0.845 & 4.164 \\
\hline DT_bagging $90 \%$ & 0.970 & 0.936 & 0.782 & 0.453 & 0.774 & 3.914 \\
\hline DT_bagging $100 \%$ & 0.976 & 0.910 & 0.742 & 0.516 & 0.777 & 3.921 \\
\hline Gradient Boosting & 0.936 & 0.941 & 0.869 & 0.768 & 0.883 & 4.398 \\
\hline HP Forest & 0.903 & 0.963 & 0.873 & 0.505 & 0.809 & 4.053 \\
\hline \multicolumn{7}{|c|}{ Variable Treatment II } \\
\hline Model & AUROC & Specificity & Precision & Recall & Accuracy & Total \\
\hline DT_no bagging & 0.904 & 0.872 & 0.765 & 0.821 & 0.855 & 4.217 \\
\hline DT_bagging 90\% & 0.949 & 0.899 & 0.683 & 0.432 & 0.742 & 3.705 \\
\hline DT_bagging $100 \%$ & 0.955 & 0.910 & 0.742 & 0.516 & 0.777 & 3.900 \\
\hline Gradient Boosting & 0.924 & 0.915 & 0.816 & 0.747 & 0.859 & 4.261 \\
\hline HP Forest & 0.894 & 0.963 & 0.848 & 0.411 & 0.777 & 3.893 \\
\hline \multicolumn{7}{|c|}{ Variable Treatment III } \\
\hline Model & AUROC & Specificity & Precision & Recall & Accuracy & Total \\
\hline DT_no bagging & 0.910 & 0.888 & 0.781 & 0.789 & 0.855 & 4.224 \\
\hline DT_bagging 90\% & 0.946 & 0.910 & 0.691 & 0.400 & 0.739 & 3.685 \\
\hline DT_bagging $100 \%$ & 0.953 & 0.888 & 0.700 & 0.516 & 0.763 & 3.820 \\
\hline Gradient Boosting & 0.919 & 0.926 & 0.827 & 0.705 & 0.852 & 4.229 \\
\hline HP Forest & 0.888 & 0.957 & 0.840 & 0.442 & 0.784 & 3.912 \\
\hline
\end{tabular}


486 Table 2. Comparison of five performance measures among five establishment models of exotic 487 birds in Taiwan, separately for three variable treatments (see Methods for details).

Variable Treatment I

\begin{tabular}{|c|c|c|c|c|c|c|}
\hline Model & AUROC & Specificity & Precision & Recall & Accuracy & Total \\
\hline DT_no bagging & 0.839 & 0.898 & 0.806 & 0.694 & 0.821 & 4.059 \\
\hline DT_bagging $90 \%$ & 0.945 & 0.932 & 0.800 & 0.444 & 0.747 & 3.869 \\
\hline DT_bagging $100 \%$ & 0.963 & 0.949 & 0.842 & 0.444 & 0.758 & 3.957 \\
\hline Gradient Boosting & 0.985 & 1.000 & 1.000 & 0.861 & 0.947 & 4.793 \\
\hline HP Forest & 0.901 & 0.983 & 0.875 & 0.194 & 0.684 & 3.638 \\
\hline \multicolumn{7}{|c|}{ Variable Treatment II } \\
\hline Model & AUROC & Specificity & Precision & Recall & Accuracy & Total \\
\hline DT_no bagging & 0.839 & 0.898 & 0.806 & 0.694 & 0.821 & 4.059 \\
\hline DT_bagging $90 \%$ & 0.942 & 0.932 & 0.800 & 0.444 & 0.747 & 3.866 \\
\hline DT_bagging $100 \%$ & 0.963 & 0.949 & 0.842 & 0.444 & 0.758 & 3.957 \\
\hline Gradient Boosting & 0.976 & 0.983 & 0.969 & 0.861 & 0.937 & 4.726 \\
\hline HP Forest & 0.914 & 1.000 & 1.000 & 0.167 & 0.684 & 3.765 \\
\hline \multicolumn{7}{|c|}{ Variable Treatment III } \\
\hline Model & AUROC & Specificity & Precision & Recall & Accuracy & Total \\
\hline DT_no bagging & 0.839 & 0.898 & 0.806 & 0.694 & 0.821 & 4.059 \\
\hline DT_bagging $90 \%$ & 0.936 & 0.932 & 0.800 & 0.444 & 0.747 & 3.860 \\
\hline DT_bagging $100 \%$ & 0.940 & 0.949 & 0.842 & 0.444 & 0.758 & 3.934 \\
\hline Gradient Boosting & 0.971 & 1.000 & 1.000 & 0.778 & 0.916 & 4.665 \\
\hline HP Forest & 0.912 & 1.000 & 1.000 & 0.139 & 0.674 & 3.725 \\
\hline
\end{tabular}

